\title{
A tale of two cores: triggered massive star formation in the bright-rimmed cloud SFO 75
}

\author{
J. S. Urquhart ${ }^{1}$, M. A. Thompson ${ }^{2}$, L. K. Morgan ${ }^{3}$, M. R. Pestalozzi ${ }^{2}$, Glenn J. White ${ }^{4,5}$, and D. N. Muna ${ }^{6}$ \\ 1 School of Physics and Astronomy, University of Leeds, Leeds, LS2 9JT, UK \\ e-mail: jsu@ast. leeds.ac.uk \\ ${ }^{2}$ Centre for Astrophysics Research, Science and Technology Research Institute, University of Hertfordshire, College Lane, Hatfield, \\ AL10 9AB, UK \\ 3 Green Bank Telescope, PO Box 2, Green Bank, WV 24944, USA \\ 4 Dept. of Physics \& Astronomy, The Open University, Walton Hall, Milton Keynes, MK7 6AA, UK \\ 5 Space Physics Division, Space Science \& Technology Division, CCLRC Rutherford Appleton Laboratory, Chilton, Didcot, \\ Oxfordshire, OX11 0QX, UK \\ ${ }^{6}$ Dept. of Physics \& Astronomy, University of Sheffield, Hicks Building, Hounsfield Road, Sheffield, S3 7RH, UK
}

Received 5 February 2007 / Accepted 12 March 2007

\section{ABSTRACT}

\begin{abstract}
Context. Bright-rimmed clouds (BRCs) are isolated molecular clouds located on the edges of evolved HII regions. Star formation within the BRCs may have been triggered through the propagation of photoionisation-induced shocks driven by the expansion of the HII region.

Aims. The main focus of this paper is to investigate the current level of star formation within one of these clouds and evaluate to what extent, if any, star formation may have been triggered.

Methods. We present a detailed multi-wavelength study of the BRC SFO 75, including $1.3 \mathrm{~cm}$ and $1.2 \mathrm{~mm}$ continuum, and ${ }^{13} \mathrm{CO}$ and ammonia spectral line observations. To build up a comprehensive picture of the local environment we complement our observations with archival data from the 2MASS, GLIMPSE and IRAS surveys.

Results. The ${ }^{13} \mathrm{CO}$ and $1.2 \mathrm{~mm}$ emission reveals the presence of a dense core located behind the bright rim of the cloud which is approximately coincident with that of the IRAS point source. From an analysis of the IRAS and $1.2 \mathrm{~mm}$ fluxes we derive a dust temperature of $\sim 30 \mathrm{~K}$, a luminosity of $L_{\mathrm{bol}}=1.6 \times 10^{4} L_{\odot}$ and estimate the core mass to be $\sim 570 M_{\odot}$. The higher resolution ammonia observations resolve the $1.2 \mathrm{~mm}$ core into two distinct cores, one directly behind the cloud's rim (Core A) and the second located slightly farther back (Core B). These have masses of 8-15 $M_{\odot}$ and 3.5-7 $M_{\odot}$ for Core A and Core B respectively, which are significantly larger than their virial masses. Comparing the morphology of Core A with that of the photon-dominated region and ionised boundary layer leaves little doubt that it is being strongly affected by the ionisation front. 2MASS and GLIMPSE archive data which reveal a small cluster of three deeply embedded $\left(A_{\mathrm{v}} \sim 20 \mathrm{mag}\right)$ high- and intermediate-mass young stellar objects towards Core A leads us to conclude that the star formation found towards this core has been triggered. In stark contrast, Core B appears to have a much simpler, almost spherical, morphology. No stars are found towards Core B. We find evidence supporting the presence of shocked gas within the surface layers of the cloud which appears to extend to midway between the two ammonia cores.

Conclusions. The scenario that emerges from our analysis is one where the two ammonia cores pre-date the arrival of the ionisation front. Since its arrival the over-pressure of the ionised gas at the surface of the cloud has driven shocks into the surface layers of the cloud. The propagation of these shocks through Core A have triggered the formation of a small cluster of massive stars, however, the shock front has not yet propagated deeply enough into the cloud to have affected the evolution of Core B.
\end{abstract}

Key words. radio continuum: stars - stars: formation - stars: early-type - stars: pre-main sequence - ISM: clouds -

ISM: individual object: SFO 75.

\section{Introduction}

Bright-rimmed clouds (BRCs) are isolated molecular clouds located on the edges of evolved HII regions. Star formation within the BRCs may thus have been triggered through the propagation of photoionisation-induced shocks driven by the expansion of their HII regions. The photoionisation of a BRC's surface layers by UV photons from nearby OB stars leads to the formation of a layer of hot ionised gas. This is known as the ionised boundary layer (IBL), which surrounds the rim of the molecular cloud. Shocks are driven into the molecular gas if the IBL is over-pressured with respect to the molecular gas within the BRC, resulting in the compression of the cloud. This can lead to the formation of dense cores, which are then triggered to collapse by the same (or a subsequent) shock front (Elmegreen 1992). This propagating shock front may also serve to trigger the collapse of pre-existing dense cores, leading to the creation of a new generation of stars.

This mode of star formation is known as radiative-driven implosion (RDI) and may be responsible for the production of hundreds of stars in each HII region (Ogura et al. 2002), perhaps even contributing up to $\sim 15 \%$ of the low-to-intermediate mass initial mass function (Sugitani et al. 2000). In order to evaluate this mode of triggered star formation Sugitani et al. (1991) and Sugitani \& Ogura (1994) identified 89 BRCs (commonly referred to as the SFO catalogue) where star formation is likely to be taking place. These clouds were identified by correlating IRAS point sources - having colours consistent with embedded 
protostars - with clouds displaying optically bright rims from the Sharpless HII region catalogue (Sharpless 1959) and the ESO(R) Southern Hemisphere Atlas.

We have reported in an earlier paper the results of a set of radio continuum observations made towards all 45 SFO objects located in the southern hemisphere (see Thompson et al. 2004 for details; hereafter Paper I). In this paper we identified 18 BRCs that are associated with centimetre continuum emission which is spatially and morphologically consistent with the presence of an IBL (i.e., radio continuum emission is elongated parallel to the optical emission and lies in front of the cloud with respect to the direction of ionisation). Follow-up molecular line and high resolution radio continuum observations were carried out on a sample of four clouds from Paper I in order to evaluate whether the presence of the ionised gas was having a direct influence on the evolution of these clouds and the inferred star formation within them. Dense molecular cores were found to be located directly behind the ionised rims with respect to the direction of their ionising stars (Urquhart et al. 2006; hereafter Paper II). Evaluating the pressure balance between the ionised and molecular gas, we identified two clouds in approximate pressure balance and therefore likely to be in a post-shocked state, and two clouds that are in the early stages of radiative-driven collapse with shocks still being driven into them.

From Paper II we were able to identify two clouds at an early stage of interaction with the expanding ionisation front and found tentative evidence for star formation within these clouds. In this, the first of two papers, we present a detailed multiwavelength study of SFO 75, with SFO 76 being the subject of a subsequent paper (Urquhart et al. 2007, in prep.). These include radio and millimetre continuum, ${ }^{13} \mathrm{CO}$ and ammonia spectral line observations as well as 2MASS, GLIMPSE and IRAS archival data. The main focus is to investigate the current level of star formation within this cloud and evaluate to what extent, if any, the star formation may have been triggered. The structure of this paper is as follows: in Sect. 2 we summarise the main features of SFO 75 and its host HII region; in Sect. 3 we describe the observational procedures and data reduction processes; we present the observational results and analyses in Sect. 4; our main findings are discussed in Sect. 5 with particular emphasis on whether star formation has been triggered; and in Sect. 6 we present a summary of our results and highlight our main findings.

\section{SFO 75 and its environment: $\mathbf{H}_{\alpha}$ and $8 \mu \mathrm{m}$}

SFO 75 is situated on the edge of the HII region RCW 98 that is located at a heliocentric distance of $2.8 \mathrm{kpc}$. The HII region is ionised by LSS 3423, an O9.5 IV star (Yamaguchi et al. 1999) which lies at a projected distance of $\sim 0.8 \mathrm{pc}$ from the optically bright-rim of SFO 75. Embedded within this cloud is the IRAS point source 15519-5430, which is the most luminous in the SFO catalogue with an far-IR luminosity $\left(L_{\mathrm{FIR}}\right)$ of $\sim 3.4 \times 10^{4} L_{\odot}$ (Sugitani \& Ogura 1994).

In Fig. 1 we present a three colour composite image of SFO 75 and the HII region within which it resides. This image has been constructed from an SuperCOSMOS $\mathrm{H}_{\alpha}$ image (blue; Parker et al. 2005), optical DSS-POSS II image (red) and the Spitzer-IRAC $8 \mu \mathrm{m}$ (green) image obtained from the GLIMPSE survey (Benjamin et al. 2003). The $\mathrm{H}_{\alpha}$ emission arises from the recombination of ionised hydrogen within the HII region. The $8 \mu \mathrm{m}$ band is dominated by emission from two polycyclic aromatic hydrocarbon (PAH) features centred at $7.7 \mu \mathrm{m}$ and $8.6 \mu \mathrm{m}$. PAHs are excited by UV photons that manage to penetrate some way into the molecular material surrounding the HII region, and thus their presence marks the interface between the ionised gas within the HII region and the surrounding molecular gas (Léger $\&$ Puget 1984), commonly referred to as a photo-dominated region (PDR).

The $8 \mu \mathrm{m}$ and $\mathrm{H}_{\alpha}$ emission regions can clearly be seen to be anti-correlated with each other except at the position of the BRC. The $8 \mu \mathrm{m}$ emission forms a continuous unbroken, approximately circular, ring (green) which completely surrounds the ionised gas (blue), indicating that the HII region is ionisation bounded. The roughly circular appearance of the HII region would suggest that it has expanded into a fairly homogeneous, spherically symmetric, environment. In addition to the circular ring of emission, the $8 \mu \mathrm{m}$ emission reveals the presence of a dust lane running from the north-east to the south-west at an angle of $\sim 45^{\circ}$, bisecting the HII region giving it a "coffee bean" like appearance.

SFO 75 stands out dramatically against the otherwise classical looking HII region. In Fig. 1 the bright rim is seen as a thin white region that curves slightly away from the ionising star. The thin rim seen in the optical plate is consistent with the cloud and the illuminating star being located either in the same plane of the sky, or with the cloud being located slightly in the foreground. However, the cloud cannot be too far in the foreground as we would expect to see a region of extinction in the $\mathrm{H}_{\alpha}$ emission from the dense gas within the cloud, and this is not observed. We therefore conclude that SFO 75 protrudes into the HII region and lies approximately in the same plane as the ionising star, and thus its projected distance from the ionising star is likely to be close to its true distance from it.

\section{Observations and data reduction procedures}

\subsection{ATCA centimetre continuum and ammonia observations}

Observations of the ammonia $(1,1)$ inversion transition and the $1.3 \mathrm{~cm}$ radio continuum were made on the 29th April $2004 \mathrm{using}$ the Australia Telescope Compact Array (ATCA), which is located at the Paul Wild Observatory, Narrabri, New South Wales, Australia $^{1}$. The ATCA consists of $6 \times 22 \mathrm{~m}$ antennas, 5 of which lie on a $3 \mathrm{~km}$ east-west railway track with the sixth antenna located $3 \mathrm{~km}$ farther west. This allows the antennas to be positioned in several configurations with maximum and minimum baselines of $6 \mathrm{~km}$ and $30 \mathrm{~m}$ respectively.

The observations were made at two different frequency bands centred at 23569 and $23695 \mathrm{MHz}\left(\mathrm{NH}_{3}(1,1)\right)$ using bandwidths of 128 and $8 \mathrm{MHz}$ respectively. The $8 \mathrm{MHz}$ bandwidth was divided into 512 channels, giving a frequency resolution of $15.6 \mathrm{kHz}$ and a velocity resolution of $0.2 \mathrm{~km} \mathrm{~s}^{-1}$ per channel. The observations were made over a twelve hour period providing good hour angle coverage. To correct for fluctuations in the phase and amplitude caused by atmospheric and instrumental effects the total integration time was split into blocks of $\sim 15 \mathrm{~min}$ of on-source integration sandwiched between two minute observations of the phase calibrator 1613-586. For absolute calibration of the flux density Mars was observed once during the observations for approximately ten minutes. To calibrate the bandpass the bright point source 1253-055 was also observed once during the observations. The main observational parameters are summarised in Table 1.

The calibration and reduction of the data were performed using the MIRIAD reduction package (Sault et al. 1995) following standard ATCA procedures. The task UVLIN was used to

1 The ATCA is funded by the Commonwealth of Australia for operation as a National Facility managed by CSIRO. 


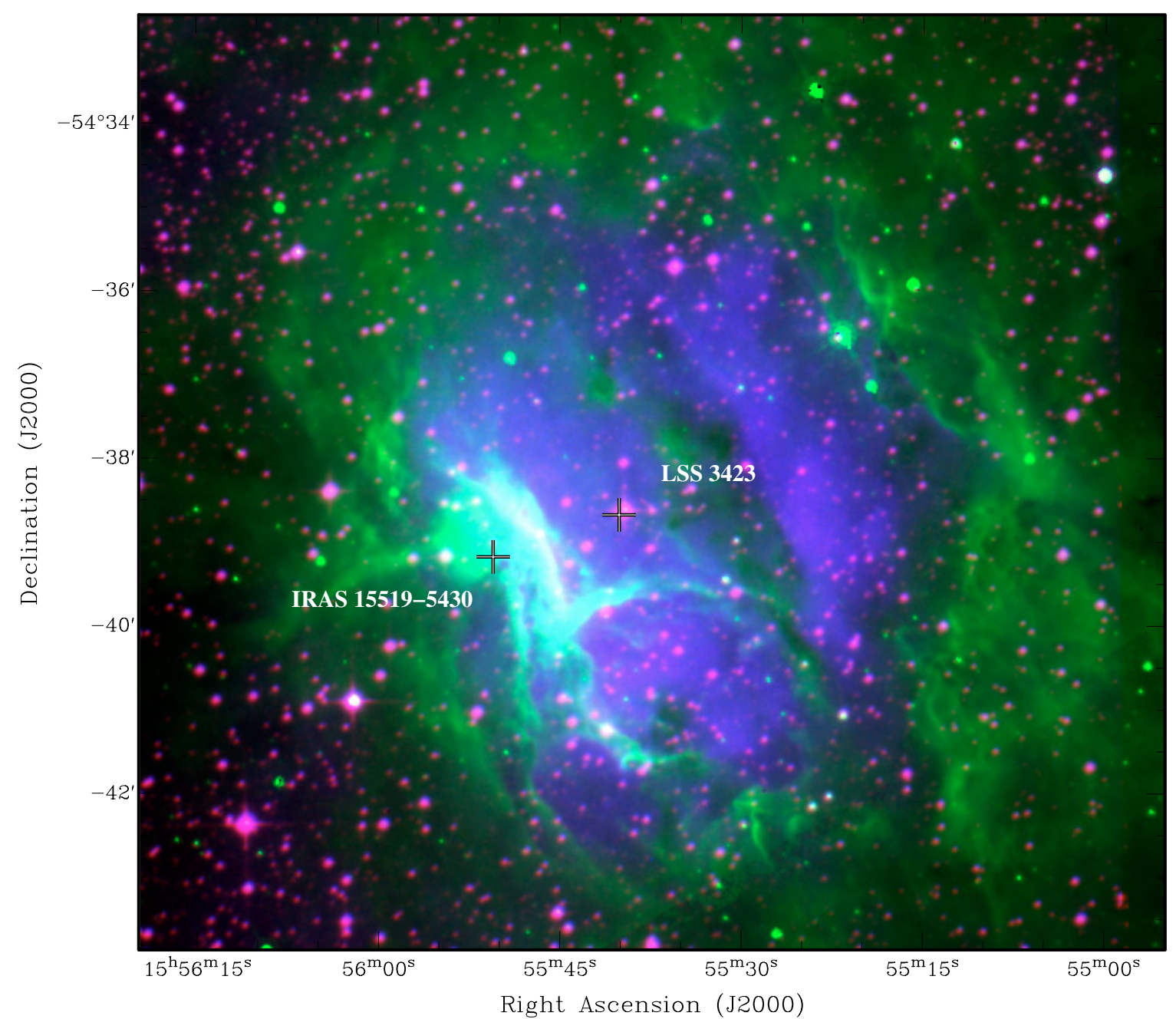

Fig. 1. Three colour composite image of SFO 75 and its associated HII region, RCW 98. The position of the IRAS point source and ionising star are indicated by a black and white cross. This composite image has been produced using a combination of the DSS red band optical image, SuperCOSMOS $\mathrm{H}_{\alpha}$ image and GLIMPSE $8 \mu \mathrm{m}$ image which are coloured red, blue and green respectively.

Table 1. Observational parameters for the ATCA $\mathrm{NH}_{3}$ and $\mathrm{cm}$ observations.

\begin{tabular}{lcc}
\hline \hline Parameter & $\mathrm{NH}_{3}(1,1)$ & $1.3 \mathrm{~cm}$ continuum \\
\hline Rest frequency $(\mathrm{MHz})$ & 23695 & 23569 \\
Total bandwidth $(\mathrm{MHz})$ & 8 & 128 \\
Primary beam $(\mathrm{arcmin})$ & 2.5 & 2.5 \\
Number of channels & 512 & 32 \\
Channel resolution $(\mathrm{kHz})$ & 15.6 & - \\
Velocity resolution $\left(\mathrm{km} \mathrm{s}^{-1}\right)$ & 0.2 & - \\
Date of observations & \multicolumn{2}{c}{29 April 2004 } \\
Total integration time (h) & \multicolumn{2}{c}{12} \\
Phase centre & $\mathrm{RA}(\mathrm{J} 2000)$ & Dec $(\mathrm{J} 2000)$ \\
& $15^{\mathrm{h}} 55^{\mathrm{m}} 49^{\mathrm{s}}$ & $-54^{\circ} 39^{\prime} 13^{\prime \prime}$ \\
Array configurations & \multicolumn{2}{c}{ EW357 } \\
Flux density calibrator & \multicolumn{2}{c}{ Mars } \\
Bandpass calibrator & \multicolumn{2}{c}{$1253-055$} \\
Phase calibrator & \multicolumn{2}{c}{$1613-586$} \\
\hline
\end{tabular}

subtract the continuum emission in the $23695 \mathrm{MHz}$ bandpass from the ammonia $(1,1)$ spectra in the $u v$ plane. These data were then CLEANed using a robust weighting of 0.5 to obtain the same sensitivity as natural weighting, but with a much improved beam-shape and lower sidelobe contamination. The CLEANed data were restored to produce an image data cube of the ammonia $(1,1)$ emission. An image of the $1.3 \mathrm{~cm}$ radio continuum emission was produced from the second frequency bandpass.

\subsection{Mopra ${ }^{13} \mathrm{CO}$ observations}

In Paper II we presented CO observations made in June 2003 towards SFO 75 with the Mopra telescope. Mopra is a $22 \mathrm{~m}$ telescope located near Coonabarabran, New South Wales, Australia. ${ }^{2}$ The telescope is situated at an elevation of $866 \mathrm{~m}$ above sea level, and at a latitude of 31 degrees south.

Emission maps were constructed from a number of single pointing observations made over a grid of positions in the ${ }^{12} \mathrm{CO}$, ${ }^{13} \mathrm{CO}$ and $\mathrm{C}^{18} \mathrm{O}(J=1-0)$ rotational lines. These observations revealed the presence of an embedded core within the rim, and allowed us to estimate its physical parameters (e.g., excitation temperature, density, mass), however, these maps proved too small $\left(2^{\prime} \times 2^{\prime}\right)$ to study the kinematics of the molecular gas. We therefore re-observed this cloud in July 2005 using the recently commissioned on-the-fly mapping mode to map the distribution

\footnotetext{
${ }^{2}$ Mopra is operated by the Australia Telescope National Facility, CSIRO and the University of New South Wales.
} 
Table 2. Observational parameters for the Mopra ${ }^{13} \mathrm{CO}$ observations.

\begin{tabular}{|c|c|c|}
\hline Parameter & & \\
\hline Rest frequency $(\mathrm{GHz})$ & \multicolumn{2}{|c|}{110.201} \\
\hline Total bandwidth (MHz) & \multicolumn{2}{|c|}{64} \\
\hline Number of channels & \multicolumn{2}{|c|}{1024} \\
\hline Velocity resolution $\left(\mathrm{km} \mathrm{s}^{-1}\right)$ & \multicolumn{2}{|c|}{0.17} \\
\hline Beam size $(")$ & \multicolumn{2}{|c|}{34} \\
\hline Date of observations & \multicolumn{2}{|c|}{ 7th July 2005} \\
\hline Total integration time (hr) & \multicolumn{2}{|c|}{$\sim 1.5$} \\
\hline Obs. positions & RA(J2000) & $\operatorname{Dec}(\mathbf{J} 2000)$ \\
\hline Map centre & $15^{\mathrm{h}} 55^{\mathrm{m}} 49.3^{\mathrm{s}}$ & $-54^{\circ} 39^{\prime} 13^{\prime \prime}$ \\
\hline Offset position & $16^{\mathrm{h}} 02^{\mathrm{m}} 17.6^{\mathrm{s}}$ & $-55^{\circ} 19^{\prime} 12^{\prime \prime}$ \\
\hline
\end{tabular}

of ${ }^{13} \mathrm{CO}$ in a $5^{\prime} \times 5^{\prime}$ region centred on the embedded core identified from our previous observations.

The receiver is a cryogenically cooled ( $\sim \mathrm{K})$, low-noise, Superconductor-Insulator-Superconductor (SIS) junction mixer with a frequency range between $85-116 \mathrm{GHz}$, corresponding to a half-power beam-width of $36^{\prime \prime}-33^{\prime \prime}$ (Mopra Technical Summary $^{3}$ ). The receiver can be tuned to either single or double side-band mode. The incoming signal is separated into two channels using a polarisation splitter, each of which can be tuned separately allowing two channels to be observed simultaneously. The receiver backend is a digital autocorrelator capable of providing two simultaneous outputs with an instantaneous bandwidth between 4-256 MHz.

The observational setup for our ${ }^{13} \mathrm{CO}$ map is summarised in Table 2. For observations of SFO 75 both polarisations were tuned to the ${ }^{13} \mathrm{CO}$ frequency, with the second channel being periodically tuned to $86.2 \mathrm{GHz}$ ( $\mathrm{SiO}$ maser frequency) to check the telescope pointing at hourly intervals. The pointing offsets were found to be $\leq 10^{\prime \prime} \mathrm{rms}$. A total of 35 rows were scanned to produce the map with an off-source position being observed at the completion of each row to allow subtraction of sky emission. Each row was scanned at a speed of $\sim 3.2 \operatorname{arcsec~s}{ }^{-1}$ with the data being averaged over a $2 \mathrm{~s}$ cycle time. Individual rows were separated by $9^{\prime \prime}$. To correct the measured antenna temperatures $\left(T_{\mathrm{A}}^{*}\right)$ for atmospheric absorption, ohmic losses and rearward spillover, a measurement was made of an ambient load (assumed to be at $290 \mathrm{~K}$ ) following the method of Kutner \& Ulich (1981) approximately every $15 \mathrm{~min}$. System temperatures were found to be stable during the observations; $T_{\text {sys }} \sim 500 \mathrm{~K}$ with variations of no more than $10 \%$ during the map of SFO 75. Absolute calibration was performed by comparing measured line temperatures of Orion KL and M17SW to standard values. We estimate the combined calibration uncertainties to be no more than $20 \%$.

The data were reduced using LIVEDATA and GRIDZILLA packages available from the ATNF. LIVEDATA performs a bandpass calibration for each row using the off-source data followed by fitting a user-specified polynomial to the spectral baseline. GRIDZILLA grids the data to a user-specified weighting and beam parameter input. The data were weighted using the periodic $T_{\text {sys }}$ measurements and a cell size of $12^{\prime \prime}$ was used to grid the data.

\subsection{SEST millimetre continuum observations}

SFO 75 and its host HII region were observed in August 2002 using the 37-channel bolometer array SIMBA (SEST Imaging Bolometer Array) at the Swedish ESO Submillimetre Telescope (SEST). SIMBA operates at a central frequency of $250 \mathrm{GHz}$

\footnotetext{
3 Available at http://www . narrabri.atnf.csiro.au/mopra/
}

which corresponds to a wavelength of $1.2 \mathrm{~mm}$, with a main beam efficiency of 0.5 and bandwidth of $50 \mathrm{GHz}$. The beam has a half power beam width of $\sim 24^{\prime \prime}$ for a single element with a sky separation of $44^{\prime \prime}$ between elements ${ }^{4}$.

An area of approximately $800 \operatorname{arcsec}^{2}$ surrounding the IRAS point source associated with SFO 75 was mapped using the fast mapping mode of SIMBA (for details see Weferling et al. 2002; Reichertz et al. 2001). Two maps of 30 min' duration and with a scan speed of $80 \operatorname{arcsec~s}^{-1}$ were coadded to make the final map. The data were reduced following the standard procedures of the MOPSI reduction package, which was written by Robert Zylka (Grenoble Observatory) ${ }^{5}$. Atmospheric extinction was measured by skydips before and after each map, with zenith opacities at $225 \mathrm{GHz}$ of typically 0.3 . Pointing was checked on an hourly basis and was found to be within $5^{\prime \prime}$. Absolute flux calibration was carried out by measurements of the $1.2 \mathrm{~mm}$ flux of Uranus. The final rms noise level in the map was found to be $\sim 20 \mathrm{mJy} \mathrm{beam}^{-1}$ and the calibration uncertainty is estimated to be $\sim 10 \%$.

\section{Results and analysis}

\subsection{Image analysis}

We present the images obtained from our observations in Fig. 2. The centimetre continuum emission can clearly be seen to form a thin layer that is elongated parallel to the optical rim and located on the outside edge of the PDR, thus confirming the presence of an IBL between the HII region and the molecular cloud. The ${ }^{13} \mathrm{CO}$ emission traces the molecular gas which is concentrated behind the bright rim falling off steeply in the direction of the $\mathrm{HII}$ region. The lowest ${ }^{13} \mathrm{CO}$ contour is almost parallel with the major axis of the IBL and the bulk of the molecular material is elongated parallel to the bright rim and perpendicular to the direction of ionisation, consistent with the hypothesis that the cloud is being compressed by the ionisation front and its associated shocks.

The $1.2 \mathrm{~mm}$ continuum emission appears to be elongated parallel to the ionisation front with a compact core of bright emission located almost directly behind the optical rim. This emission arises from a combination of optically thin thermal dust emission and thermal free-free emission from ionised gas. It is therefore possible that a significant proportion of the observed emission does not arise from the presence of an embedded core but from ionised gas associated with the bright rim. However, we can estimate the contribution from ionised gas at $1.2 \mathrm{~mm}$ by extrapolating from the integrated $1.3 \mathrm{~cm}$ emission $(30.3 \mathrm{mJy}$; see Paper II for details) assuming a thermal spectral index, i.e., $v^{-0.1}$, which we find to be $\sim 24 \mathrm{mJy}$. This is less than one per cent of the total integrated emission of 7.1 Jy. The contribution to the total flux from the thermal free-free emission is negligible and therefore the measured flux predominately arises from thermal dust emission. The $1.2 \mathrm{~mm}$ emission has a similar distribution to that of the ${ }^{13} \mathrm{CO}$ emission, although not as extended and peaks closer to the bright rim than the ${ }^{13} \mathrm{CO}$ emission $\left(\sim 26^{\prime \prime}\right)$, possibly indicating that the SIMBA observations are sampling warmer material located closer to the rim. The ${ }^{13} \mathrm{CO}$ and dust emission peaks appear to be centrally condensed and are located slightly south of the IRAS position.

\footnotetext{
4 For more details see http://www.ls.eso.org/lasilla/ Telescopes/SEST/html/telescope-instruments/simba/ index.html

5 See also http: //wWw . astro . ruhr-uni-bochum.de/nielbock/ simba/mopsi.pdf
} 
Table 3. Image parameters and peak positions of detected cores.

\begin{tabular}{lcccccc}
\hline \hline Core tracer & \multicolumn{2}{c}{ Beam parameters } & \multicolumn{2}{c}{ Position } & IRAS offset & Deconvolved core \\
& maj $\times \min \left(\left(^{\prime \prime}\right)\right.$ & $P A\left({ }^{\circ}\right)$ & $R A(J 2000)$ & $\operatorname{Dec}(\mathrm{J} 2000)$ & $\left({ }^{\prime \prime}\right)$ & $F W H M\left({ }^{\prime \prime}\right)$ \\
\hline${ }^{13} \mathrm{CO}$ & 33 & $\ldots$ & $15^{\mathrm{h}} 55^{\mathrm{m}} 50.9^{\mathrm{s}}$ & $-54^{\circ} 39^{\prime} 26^{\prime \prime}$ & 28 & 41 \\
$1.2-\mathrm{mm}$ & 24 & $\ldots$ & $15^{\mathrm{h}} 55^{\mathrm{m}} 48.7^{\mathrm{s}}$ & $-54^{\circ} 39^{\prime} 8.4^{\prime \prime}$ & 18 & 36 \\
$\mathrm{NH}_{3}$ (Core A) & $11.5 \times 4.9$ & -9.2 & $15^{\mathrm{h}} 55^{\mathrm{m}} 47.5^{\mathrm{s}}$ & $-54^{\circ} 39^{\prime} 10.2^{\prime \prime}$ & 27 & 10.4 \\
$\mathrm{NH}_{3}$ (Core B) & $11.5 \times 4.9$ & -9.2 & $15^{\mathrm{h}} 55^{\mathrm{m}} 49.5^{\mathrm{s}}$ & $-54^{\circ} 39^{\prime} 16.6^{\prime \prime}$ & 20 & 6.6 \\
\hline
\end{tabular}

The higher angular resolution of the ammonia data resolves the $1.2 \mathrm{~mm}$ core into two distinct components, one that is located directly behind the bright rim (hereafter Core A), and a second which is located farther back from the rim (hereafter Core B) directly behind Core A with respect to the direction of ionisation. The face of Core A nearest to the bright rim has a flattened morphology which almost exactly matches that of the inside edge of the PDR and the radio emission. This morphological correlation clearly indicates that these three regions are interacting with each other and that the morphology of Core A is being directly influenced by the UV illumination of LSS 3423. Core B appears to have a more spherical morphology, possibly indicating that it has not so far been significantly influenced by the passage of the photoionisation-induced shocks.

In Table 3 we present a summary of the core parameters determined from the various emission maps.

\subsection{Analysis of global properties of the ionised and molecular gas}

In Paper II we presented a detailed analysis of the internal and external gas found towards SFO 75 using our previous CO and $1.3 \mathrm{~cm}$ radio continuum observations, we therefore only provide a summary of the relevant findings here. From our $\mathrm{CO}$ analysis we found that SFO 75 has a velocity of $-37.5 \mathrm{~km} \mathrm{~s}^{-1}$, a core kinetic temperature of $\sim 24 \mathrm{~K}$, a particle density of $n=3.4 \times$ $10^{4} \mathrm{~cm}^{-3}$ and a core mass of $M_{13} \mathrm{CO}=177 M_{\odot}$. Analysis of the ionised gas determined the peak ionising photon flux to be $260 \times 10^{8} \mathrm{~cm}^{-2} \mathrm{~s}^{-1}$ and electron density to be $n_{\mathrm{e}} \sim 839 \mathrm{~cm}^{-3}$. We estimated the internal and external pressures and found that the pressure of the ionised gas is approximately three times higher than the internal pressure of the embedded $\mathrm{CO}$ core.

A drawback with our previous $\mathrm{CO}$ observations was the relatively small size of the maps $\left(2^{\prime} \times 2^{\prime}\right)$ and rather coarse sampling of the data which limited our analysis, particularly with respect to investigating the kinematics of the cloud. For this reason we re-observed this cloud using the ${ }^{13} \mathrm{CO}(J=1-0)$ line, mapping a larger region $\left(5^{\prime} \times 5^{\prime}\right)$. The integrated emission is shown in the right panel of Fig. 2. In Fig. 3 we present a position-velocity diagram taken through the cloud along the star-CO core vector (as indicated in Fig. 2) starting at the position of the ionising star (north-west corner of the image) and cutting through the core, running right to left through the diagram.

This diagram reveals the molecular material to have a steep emission gradient on the surface exposed to the ionising star, and exhibits large velocity wings $\left(\sim 6 \mathrm{~km} \mathrm{~s}^{-1}\right)$ which are perpendicular to the direction of ionisation. These are indicative of shocked gas, with the blue shifted component being associated with compressed gas moving towards us and the red shifted component being associated with compressed gas moving away from us (Lefloch \& Lazareff 1994). The similarity between the position-velocity diagram predicted by the Lefloch \& Lazareff (1994) RDI model and our observations is striking (cf. with their Fig. 14a). The presence of an IBL at the surface of the cloud, detection of a dense embedded core and the large pressure difference between the two regions led us to conclude in Paper II that this cloud was likely to be in the early stages of radiatively driven collapse. This conclusion is strengthened with the detection of the shocked gas and the similarities between our observational results and the predictions of the RDI model.

The shocked gas layer can be clearly distinguished from the bulk motion of gas behind by its significantly broader velocity distribution. We are therefore able to estimate how far this shocked layer extends into the cloud from the $F W H M$ of the shocked region. We thus estimate the shocked region to be $\sim 30^{\prime \prime}$, which corresponds to a physical distance of $\sim 0.4 \mathrm{pc}$, extending from the edge of the cloud's rim to just in front of the $\mathrm{CO}$ core. Interestingly, this would place it approximately midway between the two ammonia cores as they are seen in projection.

\section{3. $1.2 \mathrm{~mm}$ emission analysis}

\subsubsection{Luminosity, dust temperature and mass of the mm core}

In Fig. 2 we presented a contoured image of the $1.2 \mathrm{~mm}$ dust emission detected towards SFO 75, which has a peak flux density of $2.0 \mathrm{Jy} \mathrm{beam}^{-1}$ and integrated flux of 7.1 Jy. In this subsection we use these flux measurements to derive the luminosity, temperature and mass of the embedded $\mathrm{mm}$ core.

It has been shown (e.g., Sridharan et al. 2002; Beuther et al. 2002) that two components are present in the spectral energy distribution (SED) of luminous YSOs: a compact hot component which dominates the IRAS $12 \mu \mathrm{m}$ and $25 \mu \mathrm{m}$ fluxes, and a more extended component arising from colder gas which dominates the IRAS $60 \mu \mathrm{m}$ and $100 \mu \mathrm{m}$ fluxes. We therefore estimate the luminosity and temperature of these components by fitting two greybody functions to the core's SED. The greybody functions have the form (e.g., Dent et al. 1998),

$F_{v}=\Omega B_{v}\left(T_{\text {dust }}\right)\left(1-\mathrm{e}^{-\tau_{v}}\right)$

where $F_{v}$ is the flux measured at a frequency $v, \Omega$ is the solid angle subtended by the cloud, $B_{v}\left(T_{\text {dust }}\right)$ is the Planck function evaluated at the dust temperature $\left(T_{\text {dust }}\right)$ and frequency $(v)$, and $\tau_{v}$ is the optical depth at frequency $v$.

The SED of the $1.2 \mathrm{~mm}$ core was derived using fluxes from the four IRAS bands and the integrated $1.2 \mathrm{~mm}$ dust emission from the SIMBA map shown in Fig. 2. The optical depth $\tau$ is parameterized in terms of a common dust emissivity in$\operatorname{dex} \beta$ so that $\tau$ can be evaluated at arbitrary frequencies from a known reference frequency $v_{\text {ref }}$ and optical depth $\tau_{\text {ref }}$, i.e., $\tau_{v}=\tau_{\text {ref }}\left(v / \nu_{\text {ref }}\right)^{\beta} . \beta$ has typical values of 1-2 for molecular clouds, however, to limit the number of free parameters, and to maintain consistency with previous work of this kind (e.g., Faúndez et al. 2004; Sridharan et al. 2002), we have adopted a value of 1 for the hot component, but leave the emissivity of the cold component as a free parameter. We also assume that the size of the region giving rise to the cold component emission is 


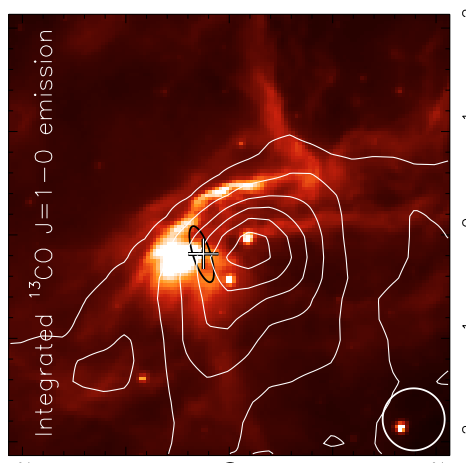

Sə7nu!N $0 X Y$
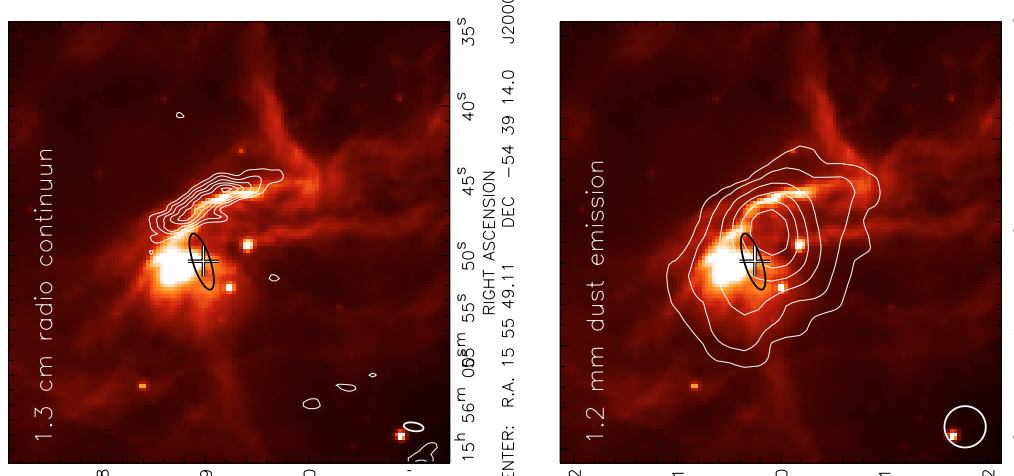

səłnuin ax

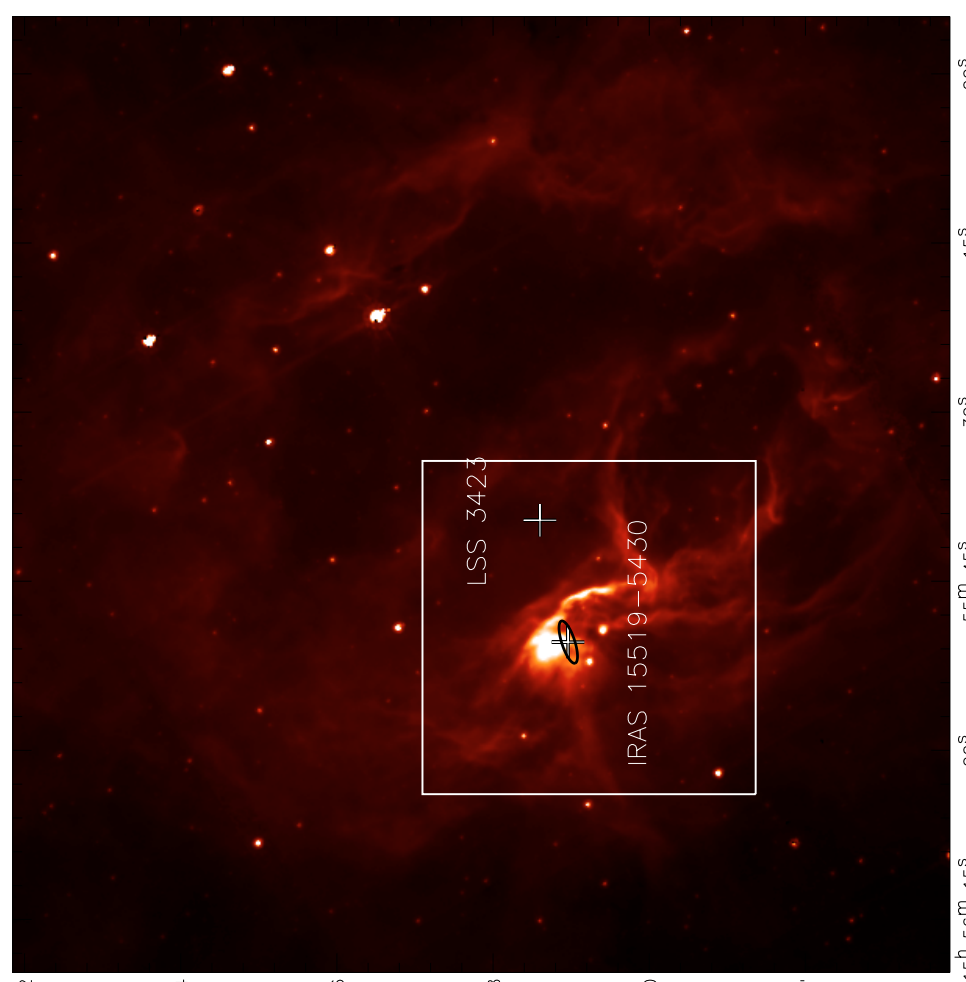

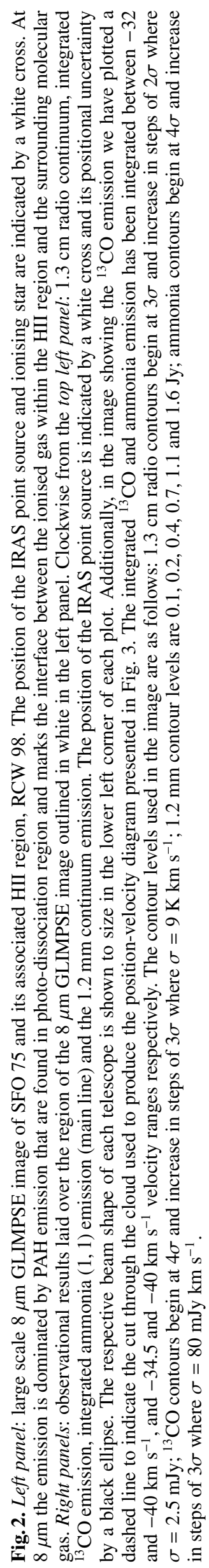




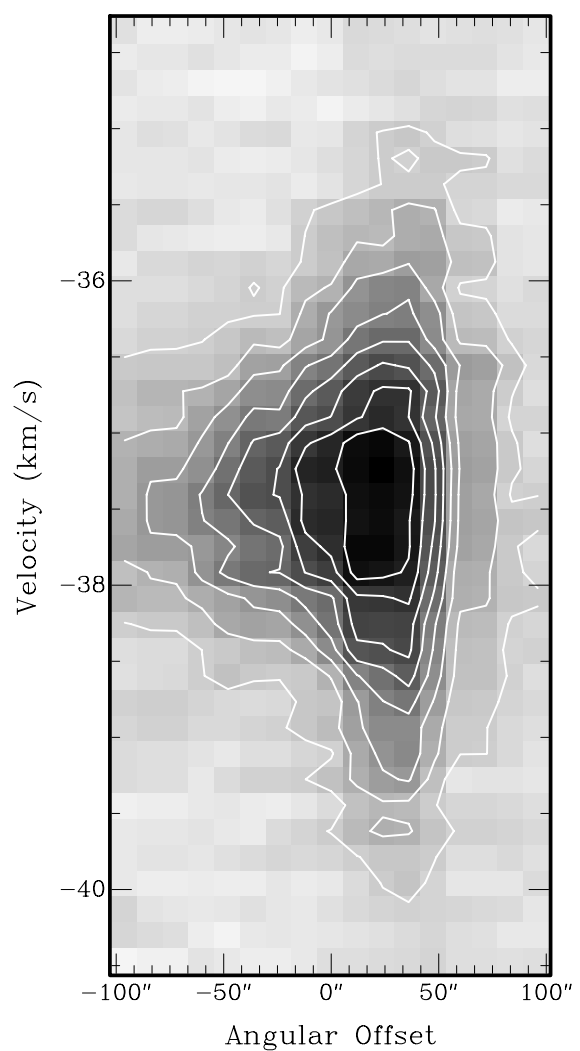

Fig. 3. Position-velocity diagram taken through the ionising star-CO core vector as indicated in Fig. 2. The direction of ionisation is from right to left. This diagram clearly reveals the presence of blue and red shifted emission at the front of the molecular cloud, indicative of shocks and the steep emission gradient consistent with compression of the cloud by the ionisation front. The contours start at $20 \%$ and increase in steps of $10 \%$ of the peak value $\left(11.1 \mathrm{~K} \mathrm{~km} \mathrm{~s}^{-1}\right)$.

the same as the $1.2 \mathrm{~mm}$ core. We leave the temperatures of the hot and cold components, and the size of the hot component as free parameters. The two component greybody fit to the SED is presented in Fig. 4; the temperatures for the hot and cold components are $175 \mathrm{~K}$ and $30 \mathrm{~K}$ respectively. We estimate the size of the hot central region to be $\sim 0.2^{\prime \prime}$ and the dust emissivity of the cold component to be $\sim 1.5$. It is the cold dust temperature that is of most interest to us as it best describes the temperature of the large scale emission traced by our $1.2 \mathrm{~mm}$ observations.

Integrating over the observed SED yields a bolometric luminosity of $\sim 1.6 \times 10^{4} L_{\odot}$, which corresponds to the presence of a single ZAMS star with a spectral class between B0 and B0.5 (Panagia 1973). Alternatively, if the observed emission is due to the presence of an embedded cluster, the luminosity of the cluster's most massive member would typically be one or two subclasses lower than assuming a single star, i.e., B2 or B3 star (Wood \& Churchwell 1989). Since the majority of the luminosity is provided by the IRAS $60 \mu \mathrm{m}$ and $100 \mu \mathrm{m}$ flux densities it is necessary to consider where the emission originates. Taking into account the size of the IRAS beam $\left(\sim 2^{\prime}\right.$ at $\left.100 \mu \mathrm{m}\right)$, which is much larger than the $\mathrm{mm}$ core, it is possible that some of the measured flux arises from the ionisation front as well as the embedded core, and therefore the derived luminosity should be considered as an upper limit. In order to better constrain the SED we attempted to obtain flux density measurements from the MIPSGAL Spitzer Legacy project ${ }^{6}$ which has recently finished

${ }^{6}$ For details see http://ssc.spitzer.caltech.edu/legacy/ abs/carey.html

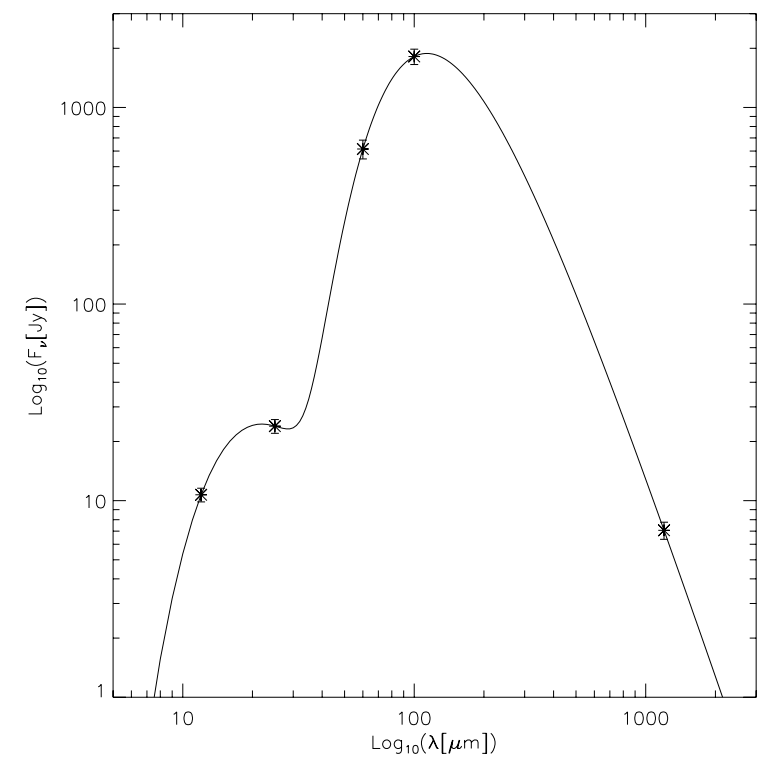

Fig. 4. Spectral energy distribution of SFO 75. Results of a two component greybody fit to the IRAS and $1.2 \mathrm{~mm}$ dust continuum emission.

surveying the GLIMPSE region at $24 \mu \mathrm{m}$ and $70 \mu \mathrm{m}$. However, this particular source lies on the edge of one of the observed fields, making it impossible to extract useful flux density measurements. Given the location of the IRAS point source and its close proximity to the mm core $\left(\sim 18^{\prime \prime}\right)$ we would suggest that the majority of the flux is associated with the core, and that the core's true luminosity is close to the upper limit.

As stated previously, the majority of the millimetre emission is due to optically thin thermal dust emission and by making a few assumptions it is possible to relate the integrated millimetre flux density to the total mass (gas+dust) of the core via (Hildebrand 1983),

$M_{\text {(gas+dust) }}=g \frac{F_{1.2 \mathrm{~mm}} D^{2}}{\kappa_{1.2 \mathrm{~mm}} B_{1.2 \mathrm{~mm}}\left(T_{\text {dust }}\right)} M_{\odot}$

where $F_{1.2 \mathrm{~mm}}$ and $\kappa_{1.2 \mathrm{~mm}}$ are the integrated flux density and dust opacity per unit mass measured at $1.2 \mathrm{~mm}$ respectively, $B_{1.2 \mathrm{~mm}}\left(T_{\text {dust }}\right)$ is the Planck function for a dust temperature $T_{\text {dust }}$, $D$ is the distance in kpc and $g$ is the gas-to-dust ratio, which we assume to be 100 . Using a value for $\kappa_{1.2 \mathrm{~mm}}=1 \mathrm{~cm}^{2} \mathrm{~g}^{-1}$ (Ossenkopf \& Henning 1994) and a value of $30 \mathrm{~K}$ for the dust temperature (as determined for the cold dust component of the greybody fit) we estimate the mass of the $\mathrm{mm}$ core to be $\sim 570 M_{\odot}$. Given the uncertainties involved in this calculation, and that of the $\mathrm{CO}$ derived mass (i.e., $M_{{ }^{13} \mathrm{CO}}=177 M_{\odot}$ ), we find them to be in reasonable agreement.

The luminosity and temperatures of the hot and cold components, which are similar to those found towards other massive embedded objects (e.g., Sridharan et al. 2002), and the derived mass leads us to conclude that massive star formation is currently taking place within SFO 75. This is consistent with the statistical trend for BRCs to preferentially form more massive stars or multiple stellar systems than found towards Bok globules and isolated dense cores (e.g., Sugitani \& Ogura 1994; Yamaguchi et al. 1999).

\subsubsection{Molecular ring surrounding the $\mathrm{HII}$ region}

The only $1.2 \mathrm{~mm}$ emission detected in our wide-field SIMBA observations was that associated with SFO 75. This implies that the 

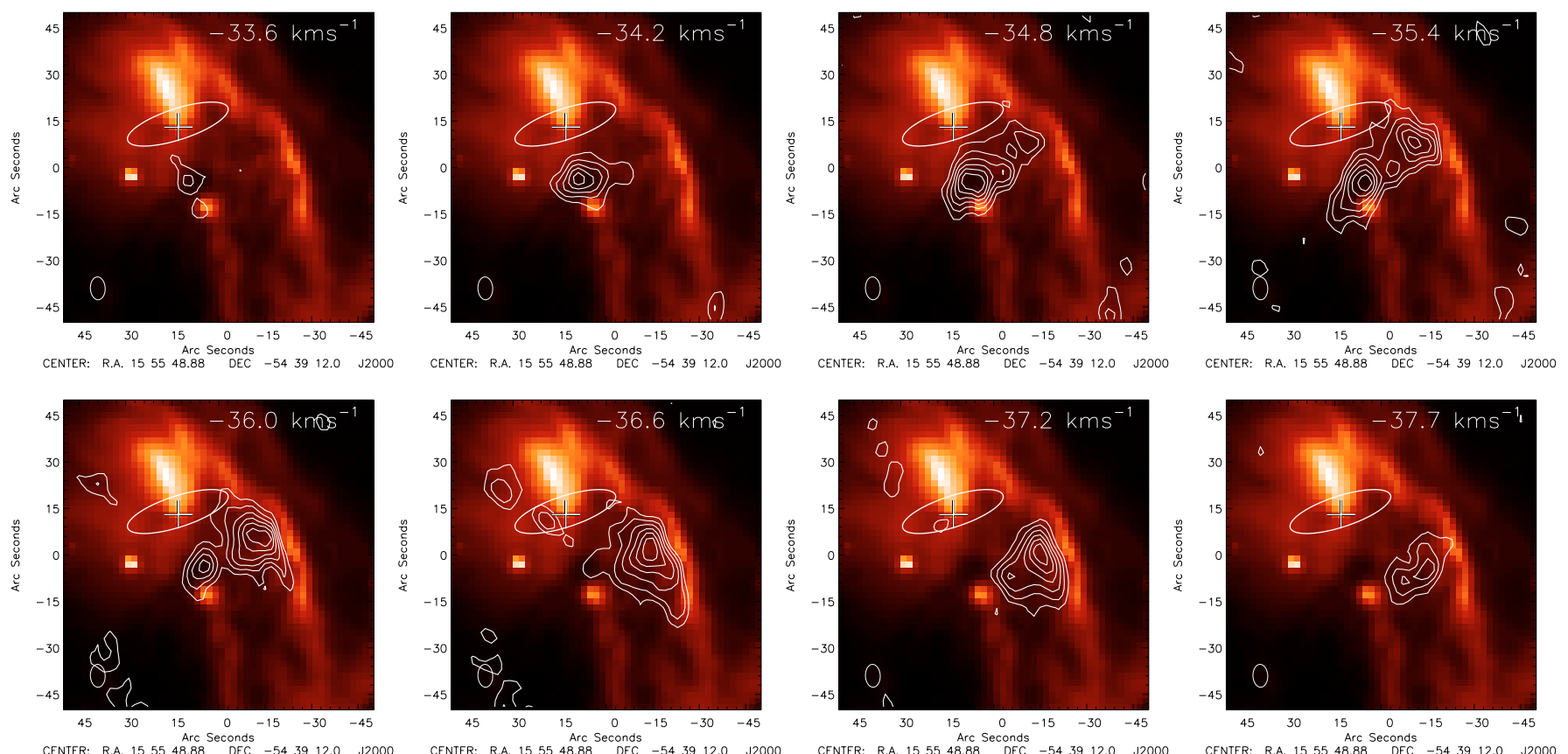

Fig. 5. Velocity channel maps of the ammonia $(1,1)$ main line emission contoured over an enlarged region of $8 \mu \mathrm{m}$ image presented in Fig. 2 (highlighted white square in lower right image). The central velocities are indicated in the top right corners of each map and are $0.6 \mathrm{~km} \mathrm{~s} \mathrm{~s}^{-1}$ thick. The position of the IRAS point source and its associated error are indicated by a cross and ellipse respectively. The first contour starts at $4 \sigma$ and increase in steps of $4 \sigma$. The size of the synthesised beam is shown to scale in the lower left corner.

molecular shell of material surrounding the HII region (inferred from the presence of the PDR traced by the $8 \mu \mathrm{m}$ PAH emission) must be of relatively low column density. We are therefore unable to determine the gas density within this ring, however, we can use the image rms to place an upper limit on the mass/beam. Assuming that the total mass is contained within a cylinder with a physical radius equal to that of the telescope beam on the sky and a length assumed to be equal to the width of the PDR seen in projection (i.e., $\sim 1 \mathrm{pc}$ ) we can estimate an upper limit for the density of the surrounding gas to be $10^{3} \mathrm{~cm}^{-3}$.

This upper limit for the $\mathrm{H}_{2}$ particle density in the ring implies that the expanding HII region has not yet swept up much of the surrounding material, either because the HII region is relatively young or that the surrounding molecular gas is of low density. The low density of the molecular shell surrounding RCW 98 suggests the "collect and collapse" process (e.g., Deharveng et al. 2005; Zavagno et al. 2006) has not occurred around RCW 98 and that SFO 75 is likely to be a preexisting molecular cloud recently exposed to the UV illumination of LSS 3423.

\subsection{Analysis of the ammonia cores}

In Fig. 5 we present eight velocity channel maps covering the velocity range between -33 to $-38 \mathrm{~km} \mathrm{~s}^{-1}$. These channel maps highlight important differences between the two ammonia cores. First, the two cores are distinct both spatially and in velocity, with Core A and Core B centred at approximately $-37 \mathrm{~km} \mathrm{~s}^{-1}$ and $-35 \mathrm{~km} \mathrm{~s}^{-1}$ respectively (see also Fig. 6). Secondly, they illustrate the excellent correlation of the morphology of the flattened forward face of Core A with that of the PDR traced by the $8 \mu \mathrm{m}$ emission - the morphologies of these two regions leaves little doubt that the ionisation front is having a dramatic influence on the evolution of this core. Additionally, the ends of the
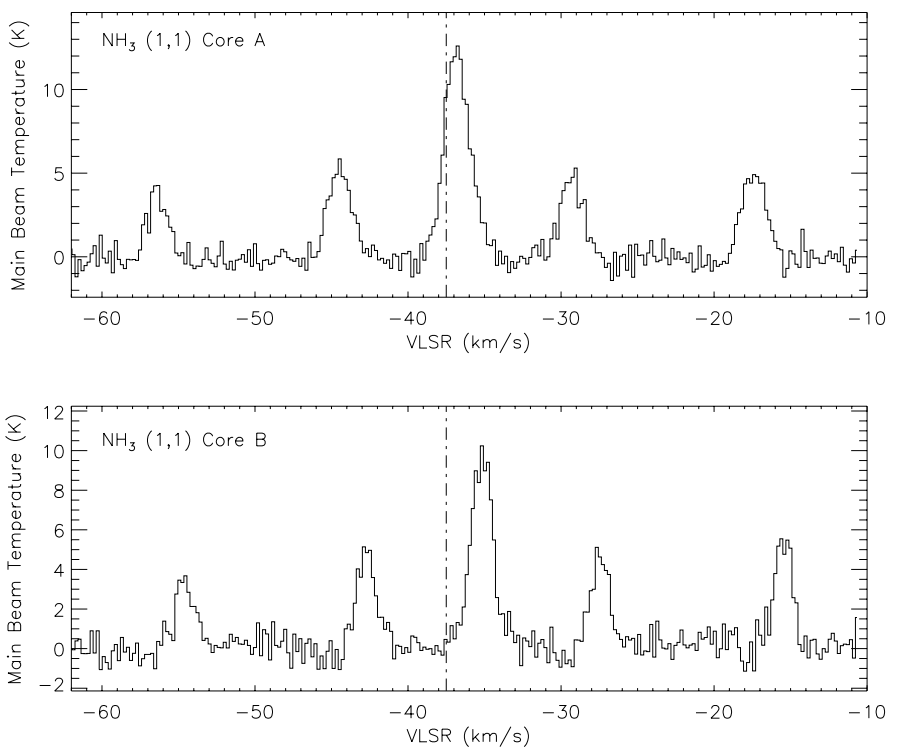

Fig. 6. Spatially integrated spectral line profiles for Core A and Core B. The vertical dashed-dotted line indicated the velocity of the ${ }^{13} \mathrm{CO}$ core.

flattened face appear to curve into the cloud, reminiscent of the small "ears" seen to develop in the Lefloch \& Lazareff (1994) model (see their Fig. 4b) where the compressed layer meets the rear edge of the initial condensation. Conversely, Core B appears to have a more spherical morphology indicating that the shock front has not yet reached it. This is confirmed by the position of the shock front which is found between the two cores A and B (see Sect. 4.2).

In Fig. 6 we present spectral line profiles integrated over each ammonia core. The hyperfine components can clearly be seen in these two spectra and can thus be used to estimate the optical 
Table 4. Fitted and derived parameters for the ammonia cores.

\begin{tabular}{ccccccccc}
\hline \hline Id & $\begin{array}{c}\text { Vel. } \\
\left(\mathrm{km} \mathrm{s}^{-1}\right)\end{array}$ & $\begin{array}{c}T_{\text {ex }} \\
(\mathrm{K})\end{array}$ & $\begin{array}{c}F W H M \\
\left(\mathrm{~km} \mathrm{~s}^{-1}\right)\end{array}$ & $\tau$ & $\begin{array}{c}N\left(\mathrm{H}_{2}\right) \\
\left(\mathrm{cm}^{-2}\right)\end{array}$ & $\begin{array}{c}n\left(\mathrm{H}_{2}\right) \\
\left(\mathrm{cm}^{-3}\right)\end{array}$ & $\begin{array}{c}\text { Mass } \\
\left(M_{\odot}\right)\end{array}$ & $\begin{array}{c}\text { Virial mass } \\
\left(M_{\odot}\right)\end{array}$ \\
\hline $\mathrm{A}$ & -37.0 & 8.0 & 1.63 & 1.41 & $4.0-7.7 \times 10^{22}$ & $0.7-1.6 \times 10^{5}$ & $8-15$ & 2.0 \\
$\mathrm{~B}$ & -35.3 & 6.4 & 1.23 & 2.58 & $4.3-8.6 \times 10^{22}$ & $1.5-3.1 \times 10^{5}$ & $3.5-7$ & 0.6 \\
\hline
\end{tabular}

depth for each core using the intensity ratio between the different hyperfine components. These profiles were fitted using the CLASS software package, in particular the METHOD $\mathrm{NH}_{3}$ utility, assuming that all of the components have equal excitation temperatures and that the line widths and separations are identical to laboratory values. Once the optical depth is known the excitation temperature of the ammonia $(1,1)$ inversion can be derived using Eqs. (1) and (2) from Harju et al. (1993)

$T_{\mathrm{B}}(1,1, m)=\eta \frac{h v}{k}\left(F\left(T_{\mathrm{ex}}\right)-F\left(T_{\mathrm{bg}}\right)\right)\left(1-\mathrm{e}^{-\tau(1,1, \mathrm{~m})}\right)$,

where the function $F(T)$ is defined as

$F(T)=\frac{1}{\mathrm{e}^{h v / k T}-1}$.

$T_{\mathrm{B}}$ is the main beam brightness temperature, $T_{\mathrm{ex}}$ is the source excitation temperature and $T_{\text {bg }}$ is the cosmic background temperature assumed to be $\sim 2.7 \mathrm{~K}, \eta$ is the beam filling factor, which we assume to be of order unity, and $m$ refers to the main hyperfine group. We have estimated the column density of molecules in the upper (1, 1) state using Eq. (3) from Harju et al. (1993),

$N_{\mathrm{u}}(1,1)=1.6 \times 10^{13} F\left(T_{\mathrm{ex}}\right) \Delta v \tau(1,1, m) \mathrm{cm}^{-2}$,

where $\Delta v$ is the $F W H M$ of the main line in $\mathrm{km} \mathrm{s}^{-1}$. To obtain the total column density for molecules in the $(1,1)$ state we need to sum the populations of both the upper and lower states. This can be done by applying the Boltzmann equation assuming both the upper and lower levels are evenly populated,

$N(1,1)=N_{\mathrm{u}}+N_{\mathrm{l}}=N_{\mathrm{u}}\left(1+\mathrm{e}^{h v / k T_{\mathrm{ex}}}\right)$.

The following equation calculates the total column density $N\left(\mathrm{NH}_{3}\right)$ making the assumption that only metastable levels are populated,

$$
\begin{aligned}
N\left(\mathrm{NH}_{3}\right)= & N(1,1)\left(\frac{1}{3} \mathrm{e}^{23.4 / T_{12}}+1\right. \\
& \left.+\frac{5}{3} \mathrm{e}^{-41.5 / T_{12}}+\frac{14}{3} \mathrm{e}^{-101.5 / T_{12}}\right) .
\end{aligned}
$$

Since there is no data available for the ammonia $(2,2)$ transition we are unable to calculate the rotational temperature, $T_{12}$. However, we are able to calculate a range of values for each parameter of interest, such as column and particle densities and core masses, using a range of rotational temperatures typical of similar cores (10-40 K; e.g., Wu et al. 2006). This range of temperatures corresponds to a difference of at most a factor of two in column density and so will not affect our results significantly. In order to obtain $\mathrm{H}_{2}$ column densities we have assumed an ammonia fractional abundance of $3 \times 10^{-8}$ (Wu et al. 2006). The $\mathrm{H}_{2}$ densities and core masses have been calculated using the core diameters presented in Table 3 . The results of the fits to the ammonia spectral profiles and core-averaged values for the derived physical parameters of the cores are presented in Table 4. We consider the masses and densities of the two cores to be lower limits due to the missing short spacings data; taking into account the uncertainties involved in their estimation they are likely to be significantly larger. Both cores appear to be centrally condensed with peak temperatures and densities a factor of $\sim 1.5$ and 2 larger, respectively, than the core-averaged values presented in Table 4 . We can test the stability of these cores by comparing their masses to their virial masses, which we calculate using the standard equation (e.g., Evans 1999),

$M_{\mathrm{vir}} \simeq 210 R_{\text {core }}\langle\Delta v\rangle^{2}$,

where $R_{\text {core }}$ is the ammonia core radius in parsecs and $\Delta v$ is the FWHM line width. The resulting virial masses for each core are presented in Table 4. Comparing these to the derived core masses reveals that both cores are significantly more massive than could be supported by their internal pressure alone (inferred from their line widths). Therefore these cores are gravitationally bound and given the large difference between their actual masses and their virial masses it is likely they are in a state of gravitational collapse.

The visual extinction can be estimated using the column density $\left(N_{\mathrm{H}_{2}}\right)$ towards each core using the following relationship: $A_{\mathrm{v}}=N_{\mathrm{H}_{2}} / 0.94 \times 10^{21} \mathrm{mag}$ (Frerking et al. 1982). This results in similar values for both cores, $A_{\mathrm{v}} \sim 42-82$ and 45-91 for Core A and Core B respectively. The visual extinction can be converted to $K$-band extinction $\left(A_{K_{\mathrm{s}}}\right)$ by multiplying by the ratio between visual and infrared extinction i.e., $A_{K_{\mathrm{s}}}=A_{\mathrm{v}} / 8.9$ (Rieke $\&$ Lebofsky 1985). In this way we obtain $K$-band extinctions between five and ten magnitudes for both cores.

\subsection{MASS and GLIMPSE point sources}

In this subsection we present complementary near- and mid-IR archival data obtained from the 2MASS and GLIMPSE surveys to investigate the stellar content of the ammonia cores. In the left panel of Fig. 7 we present a three-colour composite image produced by combining the 2MASS $H, J$ and $K_{\mathrm{s}}$ band images coloured blue, green and red respectively ${ }^{7}$. To show the spatial relationship between different regions (i.e., the dense cores, PDR and any embedded sources detected in the 2MASS data) this image has been overlaid with contours of the GLIMPSE $8 \mu \mathrm{m}$ and the integrated ammonia $(1,1)$ emission coloured orange and blue respectively. This composite image reveals the presence of a small cluster of three very red sources towards the centre of Core A (these are identified in Fig. 7 by the numbers 1, 2 and 3). None of these 2MASS sources are detected at $J$ band, and only one is detected at $H$ band, which would suggest that they are deeply embedded and that they could be protostellar in nature. The offsets from the centroid of the core position and their $J$, $H, K_{\mathrm{s}}$ magnitudes and associated quality flags are presented in Table 5.

In the right panel of Fig. 7 we present a three-colour composite image of SFO 75 produced by combining the GLIMPSE $3.6 \mu \mathrm{m}, 4.5 \mu \mathrm{m}$ and $5.8 \mu \mathrm{m}$ band images. Contours of the integrated ammonia emission are overlaid in blue. The PDR can

\footnotetext{
${ }^{7}$ Obtained from http://www.ipac.caltech.edu/2mass/
} 

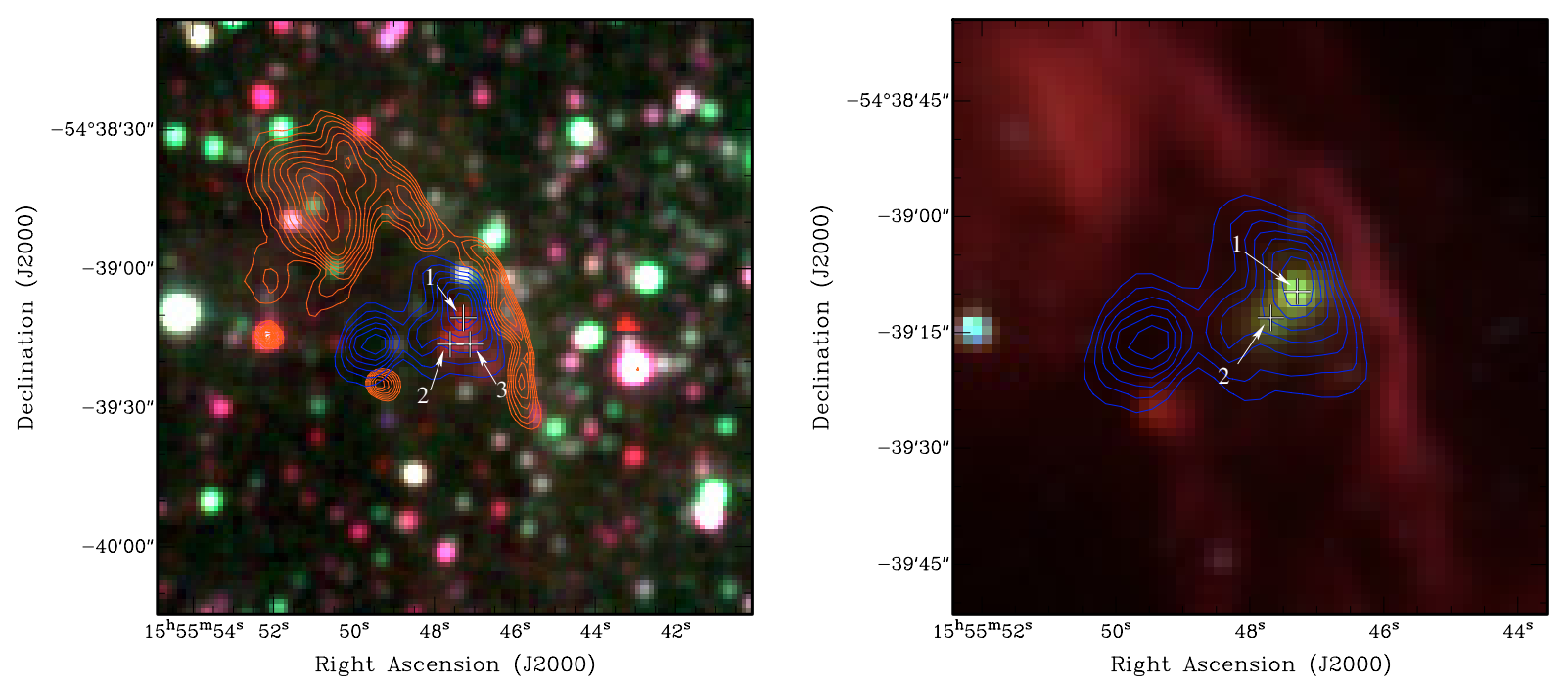

Fig. 7. $2 \mathrm{MASS}$ and GLIMPSE three colour composite images. Left panel: $2 \mathrm{MASS}$ composite produced by combining $J, H$ and $K_{\mathrm{s}}$ band images which are shown in blue, green and red respectively. This image is overlaid with contours of the GLIMPSE $8 \mu$ m emission and the integrated $\mathrm{NH}_{3}$ emission in orange and blue respectively. Right panel: GLIMPSE composite made from the 3.6, 4.5 and $5.8 \mu$ m band images which are coloured blue, green and red respectively. The positions of the 2MASS sources in both images are identified by the reference given in Table 5 .

Table 5. Parameters of red 2MASS sources located within the contoured boundary of Core A. The photometric measurements and their associated quality flags have been obtained from the 2MASS Point Source Catalogue (Cutri et al. 2003).

\begin{tabular}{|c|c|c|c|c|c|c|c|}
\hline Reference & Offset & $\overline{\mathrm{RA}}$ & Dec & $2 \mathrm{M}$ & $\mathrm{S \textrm {Ma }}$ & tudes & Quality \\
\hline ID & $(")$ & $(\mathrm{J} 2000)$ & $(\mathrm{J} 2000)$ & $J$ & $H$ & $K_{\mathrm{s}}$ & flags $^{a}$ \\
\hline 1 & 2.4 & $15: 55: 47.3$ & $-54: 39: 11.0$ & 17.1 & 14.9 & 12.8 & UUB \\
\hline 2 & 6.6 & $15: 55: 47.6$ & $-54: 39: 14.8$ & 16.8 & 14.4 & 12.8 & UUE \\
\hline 3 & 8.0 & $15: 5547.1$ & $-54: 39: 16.3$ & 16.2 & 14.6 & 13.1 & UBA \\
\hline
\end{tabular}

${ }^{a}$ These flags are as follows: (U) upper limit, (E) very poor fit, (B) $S N R>7$ and (A) $S N R>10$.

clearly be seen as a thin red rim of emission. This image reveals what appears to be an extended source which is elongated perpendicular to the rim. The peak of this emission correlates with the brightest of the detected 2MASS sources and is extended in the direction of the second brightest 2MASS source (identified as source 1 and 2 respectively in Fig. 7 and Table 5). The green colour is due to a $4.5 \mu \mathrm{m}$ excess; this band is generally dominated by stars, however, diffuse emission has been attributed to a combination of Brackett $\alpha$ and possibly $\mathrm{H}_{2}$ and $\mathrm{CO}$ in shocked regions. Diffuse $4.5 \mu \mathrm{m}$ emission has also been associated with molecular outflows - it is therefore possible that this diffuse emission is the result of ongoing star formation within Core A.

As previously mentioned, the 2MASS survey was not deep enough to have detected any of these sources at $J$ band and only one source at $H$ band. This limits our analysis of these sources and makes it impossible to use their near-IR photometric colours to determine the evolutionary state of each source (i.e., protostellar, T-Tauri or ZAMS). However, we can use the upper limits quoted for the $J$ band magnitude to derive a lower limit for spectral type and visual extinction towards each source. In Fig. 8 we present a $K_{\mathrm{s}}$ versus $J-K_{\mathrm{s}}$ magnitude-colour diagram. Three things are immediately apparent from this plot. First, two of the objects are of spectral type B2 or earlier with the third source being no later than B5. Secondly, all of the objects are embedded behind $\sim 18-23$ mag of visual extinction. This value is approximately half that calculated from the $\mathrm{H}_{2}$ column density through each core (see previous subsection) and places them roughly in the the centre of Core A. Moreover, the high values of extinction exclude the possibility that these sources are highly reddened main sequence stars located on the far side of the cloud. Finally, taking account of the 2MASS limiting $K_{\mathrm{s}}$ detection limit and assuming an average visual extinction of 20 mag the latest spectral type detectable would be a B5 ( 5.9 $M_{\odot}$; Schmidt-Kaler 1982) and therefore the cluster may have many more lower mass members that remain undetected.

The presence of two stars of spectral type B2 or earlier is in line with the findings from our mm-continuum analyses which suggests the presence of either a single high-mass star or a cluster of intermediate-mass stars. Stars with a spectral type of B3 or earlier are known to produce a significant proportion of their radiation as photons with energies high enough to ionise the surrounding material leading to the formation of an ultra-compact HII region. Therefore, we might expect to detect radio emission associated with both of the B2 or earlier stars detected. However, no radio emission has been detected towards any of the embedded sources within Core A. The non-detection of any radio emission could indicate that the embedded stars are still at a relatively early stage in their evolution, somewhat later than the hot molecular core stage, which are generally not detected at mid-IR wavelengths, but before a detectable HII region has been able to form. This is strongly supported by the detection of a methanol maser coincident with the centre of Core A recently discovered by the Methanol Multibeam (MMB) survey (MMB Consortium, private comm. $)^{8}$. Methanol masers are almost exclusively associated with the earliest stages of massive star formation turning off shortly after the formation of the UCHII region

\footnotetext{
${ }^{8}$ For more details see http://www.jb.man.ac.uk/research/ methanol/
} 


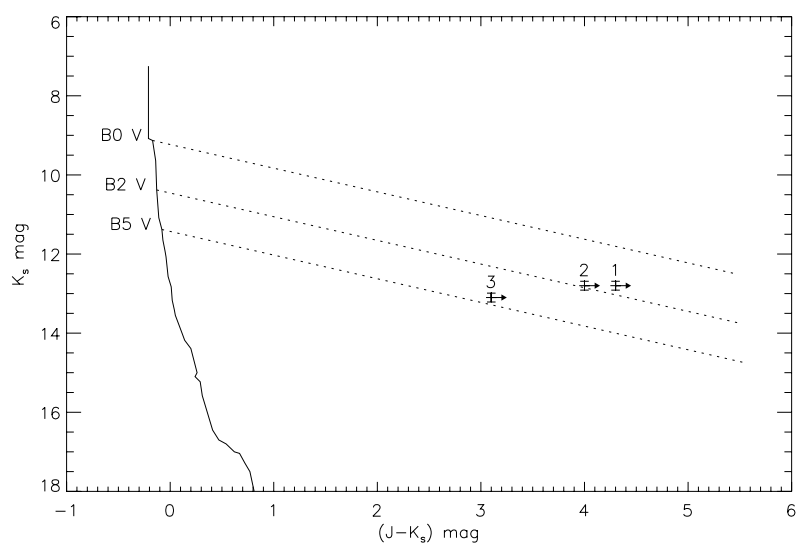

Fig. 8. $2 \mathrm{MASS} K_{\mathrm{s}}$ versus $J-K_{\mathrm{s}}$ magnitude-colour diagram of the objects detected towards Core A. The thick black line shows $K_{\mathrm{s}}$ magnitudes of main sequence stars located at a distance of $2.8 \mathrm{kpc}$, taken from Martins \& Plez (2006) and Schmidt-Kaler (1982), and assuming zero visual extinction along the line of sight. The dotted lines correspond to a visual extinction of $30 \mathrm{mag}$, which have been calculated using the standard extinction law of Mathis (1990) with $R_{\mathrm{v}}=3.1$.

(Walsh et al. 1998). The detection of maser emission towards the centre of Core A, which has a characteristic lifetime of $2.5-4.5 \times 10^{4} \mathrm{yr}$ (van der Walt 2005), allows us to place an upper limit on the age of the star formation taking place within Core A of no more than $5 \times 10^{4}$ years old. We are therefore able to conclude that these sources are deeply embedded massive young stellar objects (MYSOs).

Conversely to Core A, no 2MASS or GLIMPSE sources are seen towards Core B. The high values of extinction derived mean that we are limited to detecting only intermediate- to high-mass stars towards both cores. This makes it hard to rule out the presence of ongoing star formation within Core B completely, however, the non-detection of any stellar source from the 2MASS and GLIMPSE images does allow us to exclude the possibility that any massive star formation is taking place within Core B. If star formation is taking place within Core B it is likely to be limited to relatively low-mass stars compared to that taking place in Core A. Deeper near-IR imaging is necessary before the full stellar inventories of each core can be investigated in detail.

\section{Discussion}

There can be little doubt that the ionisation front is currently having a dramatic influence on the evolution of this cloud. The spatial distributions and morphologies of the IBL, PDR and foremost ammonia core are clearly correlated with one another, strongly suggesting these regions are interacting. In this subsection we will attempt to answer the question of whether the interaction between the ionisation front and the molecular cloud has influenced the observed star formation.

So far we have presented detailed analyses of the structure of the cloud and its associated HII region. We find compelling evidence that SFO 75 is undergoing RDI by way of an overpressured IBL and that gas dynamics indicative of shocks are being driven into the surface layers of the cloud. Additionally, we find strong evidence of recent massive star formation within a dense core located at the head of the cloud. The $1.2 \mathrm{~mm}$ data reveal the presence of a dense core located behind the cloud's rim. This core appears to be centrally condensed which would imply it is gravitationally bound or contains gravitationally bound objects within it, such as YSOs. The core has a temperature of $\sim 30 \mathrm{~K}$ - significantly higher than found towards starless cores and Bok globules - which would suggest it is internally heated, further supporting the hypothesis that the core contains protostars that are warming the surrounding material. The high luminosity and mass are similar to those found towards other massive star forming regions (Sridharan et al. 2002).

The higher angular resolution of the ammonia map resolves the $1.2 \mathrm{~mm}$ core into two smaller distinct components which are aligned perpendicular to the bright rim. It is towards the foremost of these two cores that we find strong evidence supporting the conclusions reached from our $1.2 \mathrm{~mm}$ data with the detection of a small deeply embedded cluster of high- and intermediate-mass YSOs. Since there is no evidence that star formation is taking place within Core B, located farther back from the cloud's rim, it would appear that the star formation is taking place exclusively very close to the edge of the rim of the cloud within Core A. The fact that the star formation is localised to the rim and is taking place within a core that is being strongly affected by the ionisation front and its associated shocks is persuasive circumstantial evidence that the star formation has been triggered.

The non-detection of any star formation activity towards Core B may indicate that while the ionisation front has had a dramatic affect on Core A, its associated shock front has not yet propagated far enough into the cloud to have had a significant effect on the kinematics of Core B. In this regard it is interesting to note that Core $\mathrm{A}$ is marginally warmer, more massive, and has wider line widths than Core $\mathrm{B}$, in line with what would be expected if it is undergoing RDI. Moreover, we note that Core B appears to have a much simpler, almost spherical, morphology and is offset in velocity by approximately $2 \mathrm{~km} \mathrm{~s}^{-1}$ from that of Core A. This situation is similar to examples of small scale sequential star formation seen towards other BRCs (e.g., Sugitani et al. 1995; Ogura et al. 2002; Ogura 2006).

This hypothesis is supported by our analysis of the shocked layer identified from the CO data presented in Sect. 4.2 which estimated the shock had only propagated as far as the mid-point between the two ammonia cores. If the blue and red shifted emission seen in the ${ }^{13} \mathrm{CO}$ position-velocity diagram is due to the propagation of an ionisation induced shock front, we would expect the time taken for the shock to have propagated to this point to be similar to the time that the cloud has been exposed to UV illumination from LSS 3423. The shock propagation speed can be estimated using the relationship between the pre- and postshock pressures of the neutral gas (i.e., White et al. 1999),

$V_{\mathrm{s}}^{2}=\alpha \frac{\left(P_{\mathrm{s}}-P_{\mathrm{n}}\right)}{\rho_{\mathrm{n}}}$

where $P_{\mathrm{s}}$ is the pressure of the shocked gas, $P_{\mathrm{n}}$ and $\rho_{\mathrm{n}}$ are the pressure and density in the pre-shocked neutral gas respectively. The pressure of the shocked gas has been assumed to be twice that of the ionised surface layer. The value of $\alpha$ lies between 1 and 2 and using White et al's assumption that the pre- and postshocked density ratio has a range between $2-\infty$, results in a maximum error of $\sqrt{2}$ into the estimated shock velocity. Using these assumptions we derive a shock velocity of $\sim 2 \mathrm{~km} \mathrm{~s}^{-1}$; this corresponds to a propagation time for the shock of $\sim 1.6 \times 10^{5} \mathrm{yr}$.

We can estimate the amount of time the cloud has been exposed to the ionisation front by first calculating the age of the HII region and then comparing it to the time taken for the HII region to have expanded to the edge of the cloud. The boundary between the ionised gas and the surrounding molecular gas is clearly defined by the PDR which separates the two regions. We have used this interface to estimate the angular radius of 
the HII region to be $\sim 3.5^{\prime}$, corresponding to a physical radius of $2.8 \mathrm{pc}$. When the ionising star turns on, its radiation begins ionising the surrounding medium leading to the creation of a HII region that rapidly expands out to the Strömgren radius, after which point it continues to expand more slowly. Taking account of the Strömgren radius for a O9.5 star (0.3 pc and $1.5 \mathrm{pc}$ for a HII region expanding into a medium of densities of $10^{3} \mathrm{~cm}^{-3}$ and $10^{2} \mathrm{~cm}^{-3}$ respectively), and using an expansion speed of $11.4 \mathrm{~km} \mathrm{~s}^{-1}$ for the period of expansion after the Strömgren radius has been reached, we estimate the age of the HII region to be approximately $1-2.5 \times 10^{5} \mathrm{yr}$. At a projected distance of $0.8 \mathrm{pc}$ the amount of time SFO 75 has been exposed to the ionisation radiation ranges between $1-2 \times 10^{5} \mathrm{yr}$.

Comparing the ionisation time to the shock propagation time we find them to be of similar age. Although this is not conclusive proof that the shock is directly connected to the arrival of the ionising front at the surface of the cloud, our simplistic analysis does not rule out a connection and shows them to be approximately coeval. Given that the star formation almost certainly post-dates the arrival of the ionisation front, the strong evidence of shocks within the surface layers of the cloud, and the excellent morphological correlation between the IBL, PDR and flattered face of Core A we conclude that the star formation has been triggered by the photoionisation-induced shock driven into the cloud by the expanding HII region. The lack of any star formation activity within Core $B$ further strengthens the case for the star formation within Core A to have been triggered.

\section{Summary and conclusions}

We present a multi-wavelength study of the bright-rimmed cloud SFO 75 in order to evaluate whether or not the observed star formation could have been triggered by radiative-driven implosion. This study includes observations of the $1.3 \mathrm{~cm}$ and $1.2 \mathrm{~mm}$ continuum, and ${ }^{13} \mathrm{CO}(J=1-0)$ and ammonia $(1,1)$ spectral line observations. To complement our observations we have used near-, mid- and far-IR archival data obtained from the 2MASS, GLIMPSE, and IRAS archives respectively. Combining all of these data sets has enabled us to build up a comprehensive picture of this cloud and its local environment.

RCW 98 appears to be an approximately spherical HII region surrounded by a low density $\left(<10^{3} \mathrm{~cm}^{-3}\right)$ ring of molecular material. The HII region has an angular radius of $\sim 3^{\prime} .5$, corresponding to a physical radius of $\sim 2.8 \mathrm{pc}$, from which we estimate its age to be $1-2.5 \times 10^{5} \mathrm{yr}$. SFO 75 is located on the south-east side of the HII region, and approximately lies in the same plane in the sky as the ionising star. The bright rim of SFO 75 is located $\sim 0.8 \mathrm{pc}$ from the ionising star and has been exposed to the ionising radiation for $\sim 1-2 \times 10^{5} \mathrm{yr}$.

We are able to clearly identify the ionised boundary layer (IBL), the photo-dominated region and the molecular material. The excellent morphological correlation between these three leads us to conclude that there is a high degree of interaction between them. The IBL is over-pressured with respect to the molecular gas by a factor of three and it is therefore likely that photoionisation-induced shocks are currently being driven into this cloud. This is strongly supported by the detection of red and blue shifted emission which is attributed to emission from shocked gas. We estimated the shock propagation time to be $\sim 1.6 \times 10^{5} \mathrm{yr}$ and find it to be approximately coeval with the cloud's exposure to the HII region.

Our $\mathrm{CO}$ and $1.2 \mathrm{~mm}$ data reveal the presence of a dense molecular core located slightly behind the bright rim. The density and mass of the molecular gas range between $\sim 0.3-3 \times 10^{5} \mathrm{~cm}^{-3}$ and $170-570 M_{\odot}$ respectively. We find strong evidence of recent massive star formation within a dense core located at the head of the cloud. This core appears to be centrally condensed which would imply it is gravitationally bound or contains a gravitationally bound object within it. The core temperature $(\sim 30 \mathrm{~K})$ is significantly higher than found towards starless cores and Bok globules indicating the presence of an internal source of heating, such as YSOs. Moreover, the high luminosity and mass are similar to those found towards other massive star forming regions (Sridharan et al. 2002).

The $1.2 \mathrm{~mm}$ core is resolved by our high resolution ammonia observations into two distinct cores, Core A which is located directly behind the bright rim and Core B which is located farther back. Towards Core A we find a small cluster of MYSOs (two B2 and one B5 stars or earlier) which are deeply embedded behind $\sim 20 \mathrm{mag}$ of visual extinction. The upper limit for the age of this cluster, which is approximately half the estimated ionisation time, would indicate that its formation post-dates the cloud's exposure to the HII region. Due to the limited magnitude of the 2MASS observations we are unable to detect any sources that might be present that are later than B5, so these sources might just be the most massive members of a larger cluster that remains hidden. No stars are found towards Core B and given its simple morphology and that it is located farther back from the rim than Core A leads us to conclude that the shock front has not yet penetrated far enough into the cloud to have reached this core.

Although we are unable to determine conclusively whether the observed star formation has been triggered we have presented a considerable amount of circumstantial evidence which we believe strongly supports the hypothesis that the star formation has been induced. The scenario that emerges from our analysis is one where the two ammonia cores pre-date the arrival of the ionisation front. However, since its arrival the over-pressure of the ionised gas at the surface of the cloud has driven shocks into the surface layers of the cloud. The propagation of these shocks through Core A has triggered the formation of a small cluster of massive stars, however, the shock front has not yet propagated deeply enough into the cloud to have affected the evolution of Core B.

Acknowledgements. The authors would like to thank the Director and staff of the Paul Wild Observatory, Narrabri, New South Wales, Australia for their hospitality and assistance during our observations at the Compact Array and Mopra Telescope. J.S.U. is supported by a PPARC postdoctoral grant. This research would not have been possible without the SIMBAD astronomical database service operated at CDS, Strasbourg, France and the NASA Astrophysics Data System Bibliographic Services. This research makes use of data products from the Two Micron All Sky Survey, which is a joint project of the University of Massachusetts and the Infrared Processing and Analysis Center/California Institute of Technology, funded by the National Aeronautics and Space Administration and the National Science Foundation.

\section{References}

Benjamin, R. A., Churchwell, E., Babler, B. L., et al. 2003, PASP, 115, 953 Beuther, H., Walsh, A., Schilke, P., et al. 2002, A\&A, 390, 289

Cutri, R. M., Skrutskie, M. F., van Dyk, S., et al. 2003, VizieR Online Data Catalog, 2246, 0

Deharveng, L., Zavagno, A., \& Caplan, J. 2005, A\&A, 433, 565

Dent, W. R. F., Matthews, H. E., \& Ward-Thompson, D. 1998, MNRAS, 301, 1049

Elmegreen, B. 1992, in Star Formation in Stellar Systems, 381

Evans, N. J. 1999, in Science with the Atacama Large Millimeter Array (ALMA)

Faúndez, S., Bronfman, L., Garay, G., et al. 2004, A\&A, 426, 97

Frerking, M. A., Langer, W. D., \& Wilson, R. W. 1982, ApJ, 262, 590

Harju, J., Walmsley, C. M., \& Wouterloot, J. G. A. 1993, A\&AS, 98, 51 
Hildebrand, R. H. 1983, QJRAS, 24, 267

Kutner, M. L., \& Ulich, B. L. 1981, ApJ, 250, 341

Lefloch, B., \& Lazareff, B. 1994, A\&A, 289, 559

Léger, A., \& Puget, J. L. 1984, A\&A, 137, L5

Martins, F., \& Plez, B. 2006, A\&A, 457, 637

Mathis, J. S. 1990, ARA\&A, 28, 37

Ogura, K. 2006, Bull. Astron. Soc. India, 34, 111

Ogura, K., Sugitani, K., \& Pickles, A. 2002, AJ, 123, 2597

Ossenkopf, V., \& Henning, T. 1994, A\&A, 291, 943

Panagia, N. 1973, AJ, 78, 929

Parker, Q. A., Phillipps, S., Pierce, M. J., et al. 2005, MNRAS, 362, 689

Reichertz, L. A., Weferling, B., Esch, W., \& Kreysa, E. 2001, A\&A, 379, 735

Rieke, G. H., \& Lebofsky, M. J. 1985, ApJ, 288, 618

Sault, R. J., Teuben, P. J., \& Wright, M. C. H. 1995, in Astronomical Data Analysis Software and Systems IV, ed. R. A. Shaw, H. E. Payne, \& J. J. E. Hayes, ASP Conf. Ser., 77, 433

Schmidt-Kaler, T. 1982, in Landolt-Börnstein: Numerical Data and Functional Relationships in Science and Technology - New Series, Group VI, Vol. 2, ed. K. Schaifers, \& H. H. Voigt (Berlin: Springer-Verlag), 1

Sharpless, S. 1959, ApJS, 4, 257
Sridharan, T. K., Beuther, H., Schilke, P., Menten, K. M., \& Wyrowski, F. 2002, ApJ, 566, 931

Sugitani, K., \& Ogura, K. 1994, ApJS, 92, 163

Sugitani, K., Fukui, Y., \& Ogura, K. 1991, ApJS, 77, 59

Sugitani, K., Tamura, M., \& Ogura, K. 1995, ApJ, 455, L39

Sugitani, K., Matsuo, H., Nakano, M., Tamura, M., \& Ogura, K. 2000, AJ, 119, 323

Thompson, M. A., Urquhart, J. S., \& White, G. J. 2004, A\&A, 415, 627

Urquhart, J. S., Thompson, M. A., Morgan, L. K., \& White, G. J. 2006, A\&A, 450,625

van der Walt, J. 2005, MNRAS, 360, 153

Walsh, A. J., Burton, M. G., Hyland, A. R., \& Robinson, G. 1998, MNRAS, 301, 640

Weferling, B., Reichertz, L. A., Schmid-Burgk, J., \& Kreysa, E. 2002, A\&A, 383,1088

White, G. J., Nelson, R. P., Holland, W. S., et al. 1999, A\&A, 342, 233

Wood, D. O. S., \& Churchwell, E. 1989, ApJS, 69, 831

Wu, Y., Zhang, Q., Yu, W., et al. 2006, A\&A, 450, 607

Yamaguchi, R., Saito, H., Mizuno, N., et al. 1999, PASJ, 51, 791

Zavagno, A., Deharveng, L., Comerón, F., et al. 2006, A\&A, 446, 171 